\title{
Estimating The Quality of Electroconvulsive Therapy Induced Seizures Using Decision Tree and Fuzzy Inference System Classifiers
}

\author{
Alaa M. Al-Kaysi ${ }^{1,2}$ (IEEE student member), Ahmed Al-Ani ${ }^{1}$, Verònica Gálvez ${ }^{3,4}$, Colleen K. Loo ${ }^{3,4,5}$, \\ Steve Ling ${ }^{1}$ and Tjeerd W. Boonstra ${ }^{3,6}$
}

\begin{abstract}
Electroconvulsive therapy (ECT) is an effective and widely used treatment for major depressive disorder, in which a brief electric current is passed through the brain to trigger a brief seizure. This study aims to identify seizure quality rating by utilizing a set of seizure parameters. We used 750 ECT EEG recordings in this experiment. Four seizure related parameters, (time of slowing, regularity, stereotypy and post-ictal suppression) are used as inputs to two classifiers, decision tree and fuzzy inference system (FIS), to predict seizure quality ratings. The two classifiers produced encouraging results with error rate of 0.31 and 0.25 for FIS and decision tree, respectively. The classification results show that the four seizure parameters provide relevant information about the rating of seizure quality. Automatic scoring of seizure quality may be beneficial to clinicians working in this field.
\end{abstract}

\section{INTRODUCTION}

Electroconvulsive therapy (ECT) is an effective treatment for psychiatric disorders such as depression [1]. ECT is a procedure done under general anaesthesia in which small electric currents are passed through the brain, intentionally triggering a brief seizure. ECT is safe and is considered as one of the most effective treatment methods for depression [1]. Previous research has shown that ECT efficacy may depend on the placement of the stimulating electrodes and the electrical dosage used, among other treatment parameters, and that the quality of the induced seizures is related to the efficacy of ECT [2]-[5]. Well-defined criteria for seizure quality are therefore useful for the evaluation of the therapeutic effectiveness of ECT treatments.

The detection and characterisation of seizures has also been widely studied in epilepsy and several methods have been developed for the automatic detection of seizures and prediction of their onsets [6], and the existing seizure detection methods are mainly based on electroecephalography (EEG) signal analysis [7]. However, most of these methods have not been developed for ECT induced seizures, and there is hence a gap of knowledge in this area.

The first seizure prediction method was proposed in 1970 by Viglione and colleagues [8]. Recently, there has been

(1) School of Biomedical Engineering, Faculty of Engineering and Information Technology, University of Technology Sydney, Australia. (2) Control and Systems Engineering Department, University of Technology, Baghdad, Iraq. (3) The Black Dog Institute, University of New South Wales, Australia. (4) School of Psychiatry, University of New South Wales, Australia. (5) Department of Psychiatry, St George Hospital, Kogarah, Australia. (6) QIMR Berghofer, Brisbane, Australia. e-mail: (Alaa.M.Dawoodestudent.uts.edu.au, Ahmed.Al-Ani@uts.edu.au,v.galvezortiz@student. unsw.edu.au, Colleen.loodunsw.edu.au, steve.ling@uts.edu.au t.boonstra@unsw.edu.au) an increased interest in utilizing machine learning for the development of seizure prediction techniques. For example, a method is presented in [9] to predict seizure by classifying brain activity into four states: interictal (baseline), pre-ictal (pre-seizure), ictal (during the seizure), and post-ictal (after the seizure). Interictal and pre-ictal are the most significant states that can be used to predict seizure. Another seizure detection method consists of two major steps: the first one is the extraction of appropriate quantitative features from EEG data and the second step is classification of features into two classes (seizure and non-seizure) [10].

For ECT-induced seizures, a number of studies showed that the earlier mentioned seizure parameters are related to treatment efficacy; i.e., slow wave onset, amplitude, regularity, stereotypy and post ictal suppression [2], [4], [5]. In this study, we evaluate an ECT-induced seizure scoring approach based on two classification methods, namely decision tree and fuzzy inference system (FIS). The four seizure parameters were used as inputs for the two classification methods. Decision tree is a machine learning method that has the ability to discover hidden rules in data. It produced reliable results when applied to different classification problems and has the capacity to handle missing data, which is important for the decision-making process in the medical field [11]. The fuzzy inference system is also rule-based and utilizes fuzzy features, which makes it suitable for handling uncertainties that commonly exist in medical applications. Moreover, unlike other black-box classification methods, the two classification methods are considered transparent models, as they allow interpretable mapping from the inputs to the output, which is considered an advantage for a number of medical applications. The approach presented in this study is expected to help clinicians to estimate the scoring of seizure quality as an aid in evaluating the effectiveness of ECT sessions.

\section{RATING OF SEIZURE PARAMETERS AND QUALITY}

The onset of the seizure consists of two parts, the first part represents recruitment phase (amplitude gradually increases), and is followed by the second part that consists of chaotic polyspike activity, known as polyspike phase [4]. The next phase after the polyspike phase (high frequency, low amplitude) is referred to as the slow-wave phase (low frequency and high amplitude), where it represents a spike and wave activity. Slow wave phase of the ictal-EEG: is the period of high amplitude polyspike and slow-wave activity. Following this is the termination phase that starts when the slow-wave 
TABLE I

SEIZURE QUALITY SCORE

\begin{tabular}{ll}
\hline \multicolumn{2}{l}{ Seizure quality rating } \\
\hline \hline Very Poor & 1 \\
Poor & 2 \\
Average & 3 \\
Good & 4 \\
Very Good & 5 \\
\hline \hline
\end{tabular}

phase becomes irregular and is reduced in amplitude. In this phase the amplitude and frequency of the seizure are reduced until it terminates, then the EEG trace will have a post-ictal phase [4]. We focus in this study on the major seizure quality parameters. The first parameter is time to onset of slowing (TSLOW). TSLOW represents the time where the frequency (wave/sec) of the seizure starts slowing to $\leq 5 \mathrm{~Hz}$ [5], [12]. TSLOW is scored 0 if frequency does not initially exceed $5 \mathrm{~Hz}$ [13]. The second parameter is the regularity, which represents the score of predominant morphologic pattern during slow wave/spike and wave phase [12], [13]. Another parameter that reflects the seizure quality rating is stereotypy, which is scored in the range (0-3) with half scores allowed [5]. Three variables are used to identify stereotypy scoring:

- High stereotypic: The progressive from low amplitude (chaotic polyspike activity) to high amplitude (slowwave/spike activity) is obvious without the reappearance of chaotic polyspike activity.

- Not clear spike wave morphology and the chaotic polyspike may appear after the onset of the slowwave/spike and phase.

- The amplitude is changing during the slow-wave/spike and wave-phase (investigate variability). A score of 0 , 0.5 or 1 is assigned to each of the above mentioned three variables and their summation represents the stereotypy score.

Finally, the last parameter we use to rate the seizure is post-ictal suppression. Post-ictal suppression is considered an essential parameter to predict therapeutic clinical outcomes when controlled by baseline Hamilton Rating Scale for depression and mode of stimulation [14]. The rating score of suppression ranges between (0-3) [15], with half-point scores allowed [5]. We take into consideration the time length for the decline in amplitude and frequency from the last stereotypic spike/wave. Table I lists the rating of global seizure quality using (1-5) scale [16].

\section{METHOD}

\section{A. Participants}

In this experiment, we used EEG data recorded from patients were drawn from the dataset of Gálvez et al. (2016), a study of the impact of anaesthetic technique on EEG seizure quality [16]. Patients were $\geq 18$ years old and had a clinical indication for acute ECT [16]. Patients with no medication information, especially prn medication, 24 hours before the ECT session were excluded. This study was approved by the Human Research Ethics Committee of University of New South Wales. The EEG data were collected at Wesley Hospital (Kogarah, Sydney, Australia) from April 2011 to April 2013. In total 750 recordings from 84 patients were obtained.

\section{B. Electroconvulsive Therapy Procedures}

ECT was delivered using a Mecta device (MECTA Spectrum $5000 Q$, maximum output $1152 \mathrm{mC}$ Mecta Corp., Lake Oswego, OR). Anaesthesia induction was with Propofol $1 / 2 \mathrm{mg} / \mathrm{kg}$. EEG was recorded from two fronts-mastoid EEG channels; electrode-sites were cleaned adequately [16]. Expert trained clinicians scored the seizure parameters and rated seizure quality from the recorded EEG traces, using a previously described system of rating [13].

\section{EXPERIMENT}

\section{A. Prediction seizure quality}

We propose an approach that utilizes the seizure parameters scored by clinicians to estimate the seizure quality. These features are classified using two methods as shown in Fig.1. The first one is decision tree and the second one is implemented through the construction of a fuzzy inference system. The following two sections provide details of the two methods.

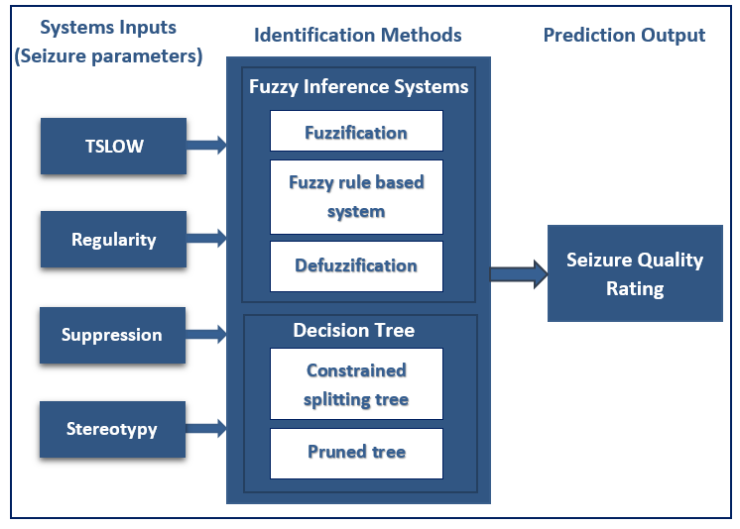

Fig. 1. Predicting model

\section{B. Decision tree classification}

A decision tree is constructed using the classification tree method. In order to reduce the tree complexity and enhance generalisation, we considered (i) enforcing a limit on the number of splits (branches and leaves) and (ii) pruning the tree using the cost-complexity pruning method that pruned the tree based on optimal pruning scheme. We used a classification error rate to select the best splitting; i.e., limits for the number of branches at the various tree levels and fixing the number of leaves to 32 . Tree-pruning aims to trim the initial branches of the tree. In general, the initial tree generated from the tree building part is large and complex, which could increase the chance of overfitting, especially if training data is noisy or contains limited samples. Pruning 


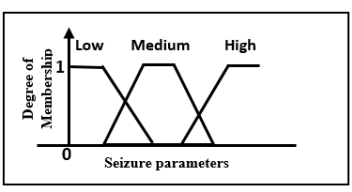

(a)

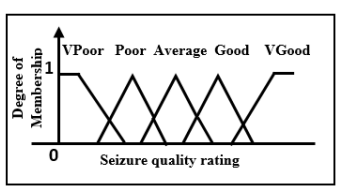

Fig. 2. Membership functions: a) fuzzy inputs, b) fuzzy output (seizure quality)

was used to improve the generalisation capability of the decision tree. An iterative approach is used in this process, which is terminated when the performance stops improving.

\section{Fuzzy inference systems FIS}

The designed fuzzy rule-based system (Mamdani) contains four inputs and one output with 11 linguistic rules. The range of the fuzzy variables (inputs and output) are: TSLOW (1$10)$, regularity (1-6), suppression (1-3), stereotypy (1-3) and seizure quality (1-5). Three different membership functions were selected for each of the four fuzzy inputs (Low, Medium and High) and five membership functions for the fuzzy output, as shown in Fig. 2. The membership functions of fuzzy output are selected based on scoring of seizure quality in Table I:

In general, the fuzzy rule-based system consists of sets of linguistic term rules in the form of IF a set of conditions for the input variables are satisfied, THEN a consequence of the output variable is inferred. In this study, we built 11 rules based on the characteristics of the input variables and their effect on seizure quality rating, as outlined in the literature. More specifically, The proposed rules are:

- Rule 1: If TSLOW is High and Stereotypy is Low, then seizure quality is Very Poor (VPoor)

- Rule 2: If Regularity is Low and Stereotypy is Medium, then seizure quality is Very Poor (VPoor)

- Rule 3: If Regularity is Medium and Stereotypy is Low and Suppression is Low, then seizure quality is Poor

- Rule 4: If Regularity is Medium and Suppression is Medium, then seizure quality is Average

- Rule 5: If Regularity is Low and Stereotypy is Medium and Suppression is Medium, then seizure quality is Average

- Rule 6: If Regularity is High and Stereotypy is Medium, then seizure quality is Good

- Rule 7: If Regularity is Medium and Stereotypy is Medium and Suppression is High, then seizure quality is Good

- Rule 8: If Regularity is High and Suppression is High, then seizure quality is Very Good (VGood)

- Rule 9: If TSLOW is Low and Regularity is High and Stereotypy is High, then seizure quality is Very Good (VGood)

- Rule 10: If TSLOW is High and Regularity is High and Suppression is Medium, then seizure quality is Good

- Rule 11: If Regularity is Medium and Stereotypy is High, then seizure quality is Very Good (VGood)
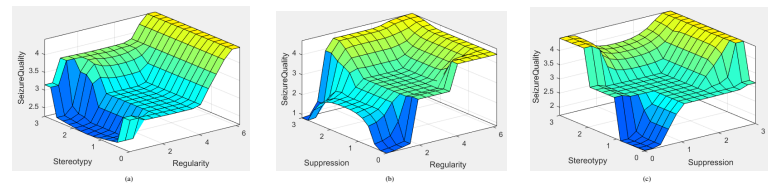

Fig. 3. Seizure quality based on (a) Stereotypy-Regularity (b) SuppressionRegularity, (c) Suppression-Stereotypy

\section{RESULTS AND DISCUSSIONS}

In order to evaluate the performance of decision tree and FIS, we used a cross-validation approach to split the data into training and testing. In the training phase, we constructed the decision tree and identified the best parameters of FIS. When provided with the seizure parameters of the 750 recordings, FIS produced 481 matches (similar scores to those recorded by the clinician experts) and 252 that differed by one point only. The scores of the remaining 17 recordings differed by two points. As the FIS provides real numbers, we calculated the error rate between the FIS output and the true scores. We found that the error rate for this case $=0.36$. Fig. 3 shows the fuzzy surface between pairs of seizure parameters and the seizure quality. The results show that there is a monotonic relationship between the seizure parameters (regularity, stereotypy and suppression) and seizure quality, which indicate that these parameters are indeed good measures for the seizure quality.

In the second case, we first predicted the seizure ratings using the fully constructed decision tree without enforcing any conditions on splitting and without applying pruning. The tree produced (105) rules and the classification results of this tree are: 520 matches, 227 differed by one score only, and the remaining 3 differed by two scores. The classification error rate is found to be 0.31 . To reduce the size of the tree and improve its classification accuracy, we used splitting and pruning techniques that resulted in noticeably simpler trees, as shown in Fig. 4. The error rates of the constrained splitting tree and pruned tree are found to be 0.30 and 0.25 respectively. Fig. 5 shows a comparison between the two prediction techniques; fuzzy inference system and decision tree (three cases; initial constrained, splitting and pruning). Table II shows the confusion matrices of FIS and three cases of decision trees. The diagonal of each matrix represents the number of correctly identified seizure quality rating, and average class-wise accuracy for two classification methods are: FIS $=0.48$ and decision tree $=0.69$. The classwise accuracy for FIS was as following: $C 1=3 / 13=$ $0.23, C 2=1 / 79=0.01, C 3=133 / 186=0.75, C 4=$ $220 / 263=0.84, C 5=124 / 208=0.60)$. Hence, this result was influenced by the bad performance of the first two classes. particularly the second one. The overall classification accuracy for two classification methods were: FIS $=0.64$, and the overall accuracy for decision tree (initial tree $=0.69$, splitting $=0.70$, and pruning $=0.76$ ). Despite the fact that decision trees produced better results compared to FIS, we believe the performance of FIS could be improved through optimization of the fuzzy rules and membership parameters. 


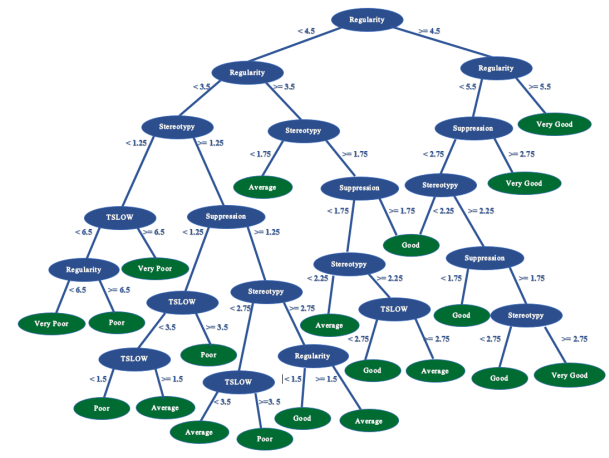

Fig. 4. Tree pruning

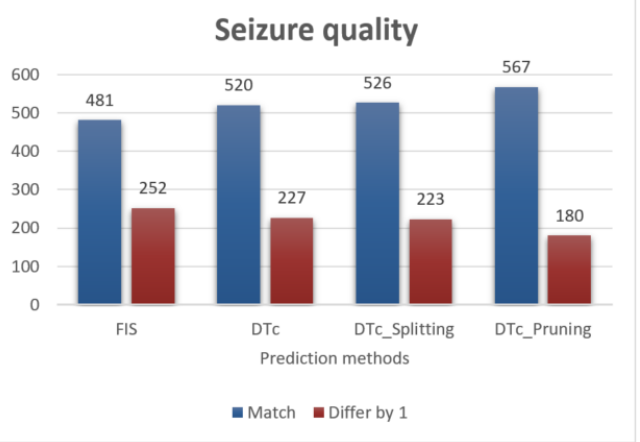

Fig. 5. Prediction seizure quality rating

More specifically, rules could be modified or weighted to enhance the accuracy of the first two classes.

TABLE II

CONFUSION MATRIX

\begin{tabular}{|lllll||lllll|}
\hline \hline \multicolumn{4}{|c||}{ Fuzzy inference system } & \multicolumn{5}{c|}{ Decision tree } \\
\hline \hline 3 & 6 & 4 & 0 & 0 & 10 & 3 & 0 & 0 & 0 \\
0 & 1 & 70 & 8 & 0 & 2 & 43 & 33 & 1 & 0 \\
0 & 2 & 133 & 51 & 0 & 0 & 19 & 133 & 34 & 0 \\
0 & 4 & 23 & 220 & 16 & 0 & 1 & 29 & 183 & 50 \\
0 & 0 & 0 & 84 & 124 & 0 & 0 & 0 & 57 & 151 \\
\hline
\end{tabular}

\begin{tabular}{|lllll||lllll|}
\hline \multicolumn{4}{|c||}{ Decision tree (Splitting) } & \multicolumn{5}{c|}{ Decision tree (Pruning) } \\
\hline \hline 10 & 3 & 0 & 0 & 0 & 10 & 3 & 0 & 0 & 0 \\
2 & 38 & 39 & 0 & 0 & 1 & 51 & 25 & 2 & 0 \\
0 & 14 & 146 & 26 & 0 & 0 & 10 & 138 & 38 & 0 \\
0 & 0 & 34 & 188 & 41 & 0 & 0 & 15 & 208 & 40 \\
0 & 0 & 0 & 64 & 144 & 0 & 0 & 0 & 48 & 160 \\
\hline
\end{tabular}

\section{CONCLUSIONS}

In this study, we identified and tested rules for automatical rating of seizure quality for ECT-induced seizures. We proposed a fuzzy rule-based inference system and a decision tree classification for this task. Rules of the fuzzy inference system (FIS) were derived from the literature through identifying relationships between the input parameters and seizure quality. We also considered using decision tree to perform this classification task, as it also provides transparent mapping from inputs to outputs. We used parameters that were rated by a team of expert clinicians as inputs to the two methods. The obtained results are encouraging in terms of achieving scores that were similar to the scores produced by expert clinicians. The proposed approach indicates that scoring of ECT induced seizures can be automated, which will be beneficial to clinicians working in this field and will provide usefuls tool for the treatment of major depressive disorder using ECT.

\section{REFERENCES}

[1] A. P. Association, The practice of electroconvulsive therapy: recommendations for treatment, training, and privileging, 2nd edn. American Psychiatric Press: Washington, DC, 2001.

[2] T. D. Perera, B. Luber, M. S. Nobler, J. Prudic, C. Anderson, and H. A. Sackeim, "Seizure expression during electroconvulsive therapy: relationships with clinical outcome and cognitive side effects," Neuropsychopharmacology, vol. 29, no. 4, p. 813, 2004.

[3] R. Weiner, C. Coffey, L. Fochtmann, R. Greenberg, K. Isenberg, C. Kellner, H. Sackeim, and L. Moench, "The practice of electroconvulsive therapy," Recommendations for Treatment, Training, and Privileging: A Task Force Report of the American Psychiatric Association, vol. 2, 2001.

[4] A. D. Krystal, R. D. Weiner, W. V. McCall, F. E. Shelp, R. Arias, and P. Smith, "The effects of ect stimulus dose and electrode placement on the ictal electroencephalogram: an intraindividual crossover study,' Biological Psychiatry, vol. 34, no. 11, pp. 759-767, 1993.

[5] M. S. Nobler, H. A. Sackeim, M. Solomou, B. Luber, D. Devanand, and J. Prudic, "Eeg manifestations during ect: effects of electrode placement and stimulus intensity," Biological psychiatry, vol. 34, no. 5, pp. 321-330, 1993

[6] A. Aarabi and B. He, "A rule-based seizure prediction method for focal neocortical epilepsy," Clinical Neurophysiology, vol. 123, no. 6, pp. 1111-1122, 2012.

[7] B. Litt and J. Echauz, "Prediction of epileptic seizures," The Lancet Neurology, vol. 1, no. 1, pp. 22-30, 2002.

[8] S. Viglione and G. Walsh, "Proceedings: Epileptic seizure prediction." Electroencephalography and clinical neurophysiology, vol. 39, no. 4 , p. $435,1975$.

[9] A. Greaves, A. Raghuvanshi, and K.-Y. Neo, "Predicting seizure onset with intracranial electroencephalogram (eeg) data-project report," 2014.

[10] S. Ramgopal, S. Thome-Souza, M. Jackson, N. E. Kadish, I. S. Fernández, J. Klehm, W. Bosl, C. Reinsberger, S. Schachter, and T. Loddenkemper, "Seizure detection, seizure prediction, and closedloop warning systems in epilepsy," Epilepsy \& behavior, vol. 37, pp. 291-307, 2014.

[11] A. Jalali, D. J. Licht, and C. Nataraj, "Application of decision tree in the prediction of periventricular leukomalacia (pvl) occurrence in neonates after heart surgery," in Engineering in Medicine and Biology Society (EMBC), 2012 Annual International Conference of the IEEE. IEEE, 2012, pp. 5931-5934.

[12] A. D. Krystal, C. E. Coffey, R. D. Weiner, and T. Holsinger, "Changes in seizure threshold over the course of electroconvulsive therapy affect therapeutic response and are detected by ictal eeg ratings," The Journal of neuropsychiatry and clinical neurosciences, vol. 10, no. 2, pp. 178186, 1998.

[13] R. D. MacPherson, J. Lawford, B. Simpson, M. Mahon, D. Scott, and C. Loo, "Low dose lignocaine added to propofol does not attenuate the response to electroconvulsive therapy," Journal of affective disorders, vol. 126, no. 1, pp. 330-333, 2010.

[14] H. Azuma, A. Fujita, K. Sato, K. Arahata, K. Otsuki, M. Hori, Y. Mochida, M. Uchida, T. Yamada, T. Akechi et al., "Postictal suppression correlates with therapeutic efficacy for depression in bilateral sine and pulse wave electroconvulsive therapy," Psychiatry and clinical neurosciences, vol. 61, no. 2, pp. 168-173, 2007.

[15] W. V. McCall, G. D. Robinette, and D. Hardesty, "Relationship of seizure morphology to the convulsive threshold." The Journal of ECT, vol. 12, no. 3, pp. 147-151, 1996.

[16] V. Gálvez, D. Hadzi-Pavlovic, H. Wark, S. Harper, J. Leyden, and C. K. Loo, "The anaesthetic-ect time interval in electroconvulsive therapy practice-is it time to time?" Brain stimulation, vol. 9, no. 1, pp. 72-77, 2016 